\title{
Overview of the Optical Design and Performance of the High Resolution Science Imaging Experiment (HiRISE)
}

\author{
Dennis Gallagher, Jim Bergstrom, Joe Day, Bryan Martin, Timothy Reed, Peter \\ Spuhler, Scott Streetman, Mark Tommeraasen, \\ Ball Aerospace and Technologies Corp. \\ Boulder, Colorado 80306
}

\begin{abstract}
The High Resolution Imaging Science Experiment (HiRISE) camera will be launched in August 2005 onboard NASA's Mars Reconnaissance Orbiter (MRO) spacecraft. HiRISE supports the MRO Mission objectives through targeted imaging of nadir and off-nadir sites with high resolution and high signal to noise ratio [a]. The camera employs a $50 \mathrm{~cm}, \mathrm{f} / 24$ all-reflective optical system and a time delay and integration (TDI) detector assembly to map the surface of Mars from an orbital altitude of $\sim 300 \mathrm{~km}$. The ground resolution of HiRISE will be $<1$ meter with a broadband red channel that can image a $6 \times 12 \mathrm{~km}$ region of Mars into a $20 \mathrm{~K} \times 40 \mathrm{~K}$ pixel image. HiRISE will image the surface of Mars at three different color bands from 0.4 to 1.0 micrometers. In this paper the HiRISE mission and its camera optical design will be presented. Alignment and assembly techniques and test results will show that the HiRISE telescope's on-orbit wave front requirement of $<0.071$ wave RMS (@633nm) will be met . The HiRISE cross track field is 1.14 degrees with IFOV $1.0 \mu$-radians.
\end{abstract}

Keywords: Optics, Mars, Optical Alignment, Camera, Telescope, Detector

\section{Introduction}

The primary functional requirement of the HiRISE camera, Figure 1, is to allow identification of both predicted and unknown features on the surface of Mars to a much finer resolution and contrast than previously possible [1], [2]. This results in a camera with a very wide swath width, $6 \mathrm{~km}$ at $300 \mathrm{~km}$ altitude, and a high signal-to-noise ratio (SNR), >100:1. Generation of terrain maps, with $30 \mathrm{~cm}$ vertical resolution, from stereo images requires very accurate geometric calibration. The project mass constraint made development of the optical system particularly challenging. In addition, the spacecraft jitter [3] must not be a major contributor to image degradation. This paper presents an overview of the HiRISE camera design and detailed discussion of the alignment and performance of the HiRISE optical system. 


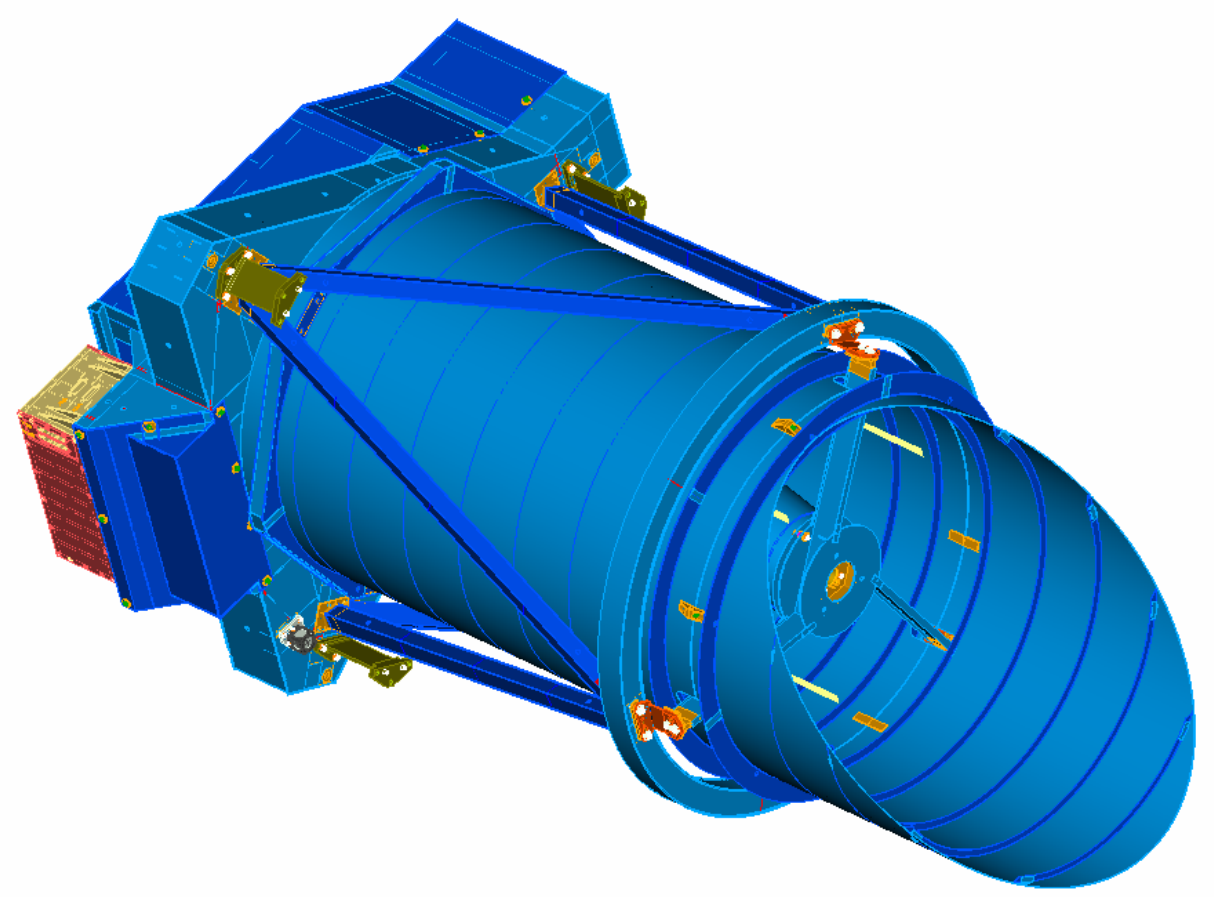

Figure 1: View of the HiRISE imager system.

\section{Instrument Overview}

The HiRISE optical requirements are shown in Table 1 and a schematic of the Optical design is shown in Figure 2. The HiRISE telescope uses a three-mirror-anastigmat optical design. The entrance aperture is $50 \mathrm{~cm}$. Two of the powered mirrors form essentially a Cassegrain telescope and the other powered mirror is an off-axis tertiary mirror that magnifies the first focus of the Cassegrain telescope to the final $f / 24$ focus (12 meters EFL). The chief ray for the HiRISE enters with a 0.45 degrees field bias. Two fold mirrors were incorporated in the optical design to allow for a more efficient light weight package since mass is a premium on the NASA MRO mission. The Fold mirror \#2 moves on a linear actuator for focus control of the HiRISE optical system. Band pass filters isolate three pass bands in the HiRISE optical system. The directions of the orbit, TDI, and field bias are shown in Figure 2.

As the nadir viewing HiRISE telescope orbits Mars the view of the detectors is scanned along the surface of Mars in a push-broom fashion. This causes the image of Mars to move across the focal plane along pixel columns. The nominal orbit for the science phase of the mission is a 3pm orbit of 255 by $320 \mathrm{~km}$ with periapsis locked to the South Pole. The ground track velocity for this orbit is approximately 3,200 m/s. Time Delayed Integration (TDI) is used to electronically transfer signal (electrons) in the 128 pixel direction at the rate the image is moving across the detectors. At $1 \times 1$ binning the TDI rate is $\sim 78$ to $110 \mu \mathrm{sec} /$ pixel. This improves signal to noise by effectively increasing the integration time by 128 times over the integration time from a single row of pixels. 
The HiRISE focal plane subsystem [4] uses 14128 x $204812 \mu$ m pixel detectors (see Figure 3. Ten of the detectors are located on the focal plane substrate in a staggered sequence to collectively form a 128 X20480 linear array forming a 1.142 degree cross track field of view. This is the Red broad band channel with a band pass of 550-850 $\mathrm{nm}$. The 128 pixel direction is compressed into one pixel via time delayed integration (TDI) to increase signal to noise. On either side of the panchromatic arrays are two pairs of detectors for blue-green and near IR imaging each formed by a pair of detectors giving a $128 \mathrm{X} 4000$ pixel array forming a 0.2 degree cross track field of view. The blue-green pass band is $400-550 \mathrm{~nm}$ and the near-IR is from $850-1000 \mathrm{~nm}$.

The HiRISE opto-mechanical structure was made from M55J graphite fiber and cyanate ester resin laminate and titanium fittings. This structure is very strong and lightweight. The entire HiRISE camera, including electronics, weighs only $65 \mathrm{~kg}$. The $50 \mathrm{~cm}$ HiRISE primary mirror weighs only $13.8 \mathrm{~kg}$.

Table 1: HiRISE Optical Requirements

\begin{tabular}{|c|c|c|c|}
\hline Diameter & \multicolumn{3}{|c|}{$50 \mathrm{~cm}$} \\
\hline EFL & \multicolumn{3}{|c|}{12000 knowledge to $1 \%$} \\
\hline Obscuration & \multicolumn{3}{|c|}{$<38 \%$} \\
\hline Field of View & \multicolumn{3}{|c|}{$\begin{array}{l}1.142 \text { cross track } \times 0.175 \text { degrees along } \\
\text { track }\end{array}$} \\
\hline Optical MTF & \multicolumn{3}{|c|}{$\begin{array}{c}>20 \% \text { MTF @ Nyquist (41.7 cycles/mm or } \\
2 \text { pixels) }\end{array}$} \\
\hline Wave Front Error & \multicolumn{3}{|c|}{$<0.071$ waves RMS @ 633 nm } \\
\hline Optical Distortion & \multicolumn{3}{|c|}{$<1 \%$} \\
\hline Spectral Bands & $\begin{array}{c}\text { Blue- } \\
\text { Gree } \\
n\end{array}$ & $\begin{array}{l}\text { Broadban } \\
\text { d Red }\end{array}$ & Near-IR \\
\hline Passband (nm) & $\begin{array}{l}400- \\
560\end{array}$ & $590-810$ & $840-1000$ \\
\hline $\begin{array}{l}\text { Passband Minimum } \\
\text { Transmission }\end{array}$ & $95 \%$ & $90 \%$ & $95 \%$ \\
\hline Stimulator Sources & \multicolumn{3}{|c|}{$\begin{array}{l}\text { Illuminate all detectors with up to } \\
\text { approximately half full well signal }\end{array}$} \\
\hline Focus Mechanism & \multicolumn{3}{|c|}{ Required for on orbit performance } \\
\hline
\end{tabular}




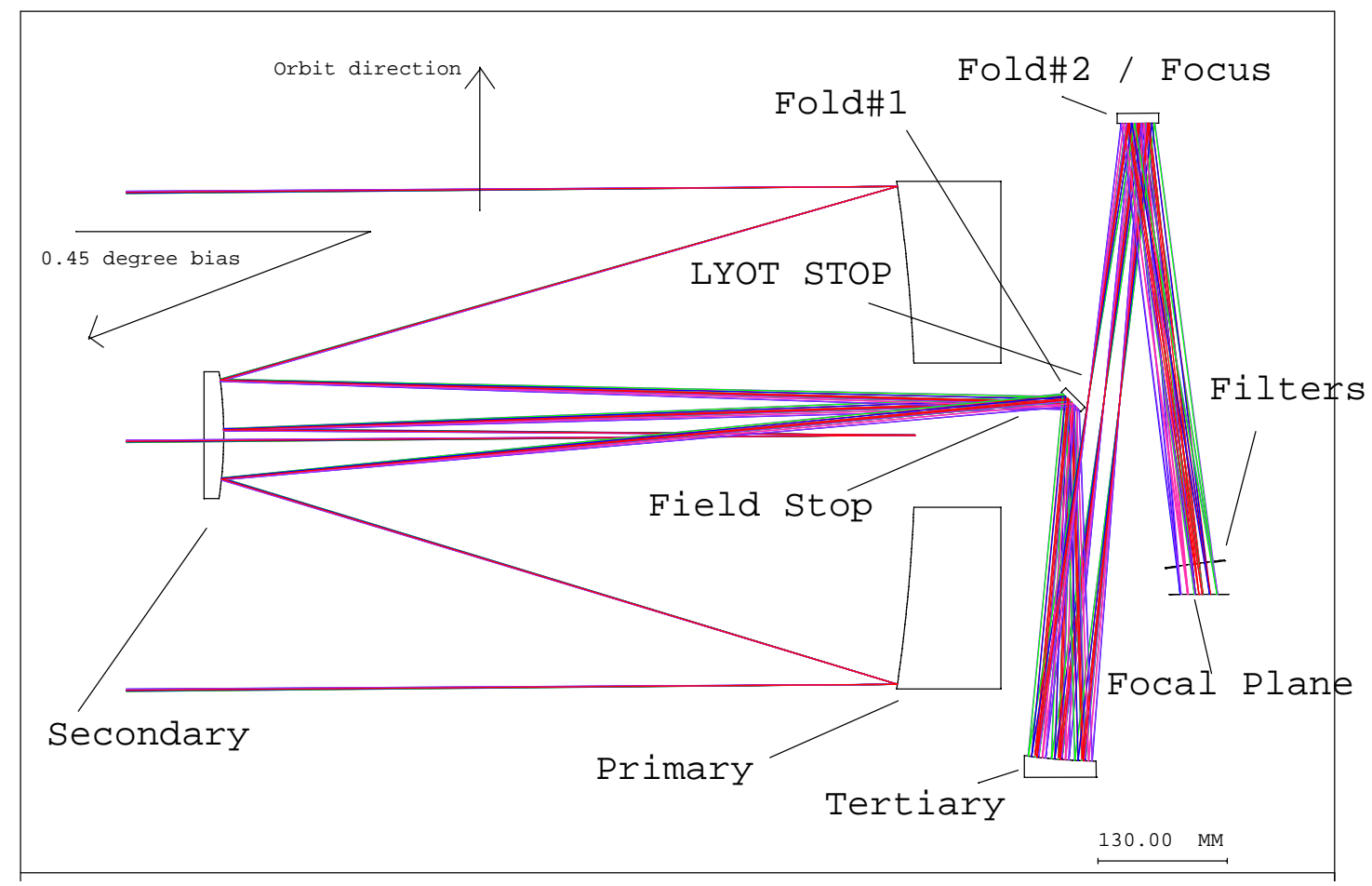

Figure 2: Cross section of the HiRISE optical design.

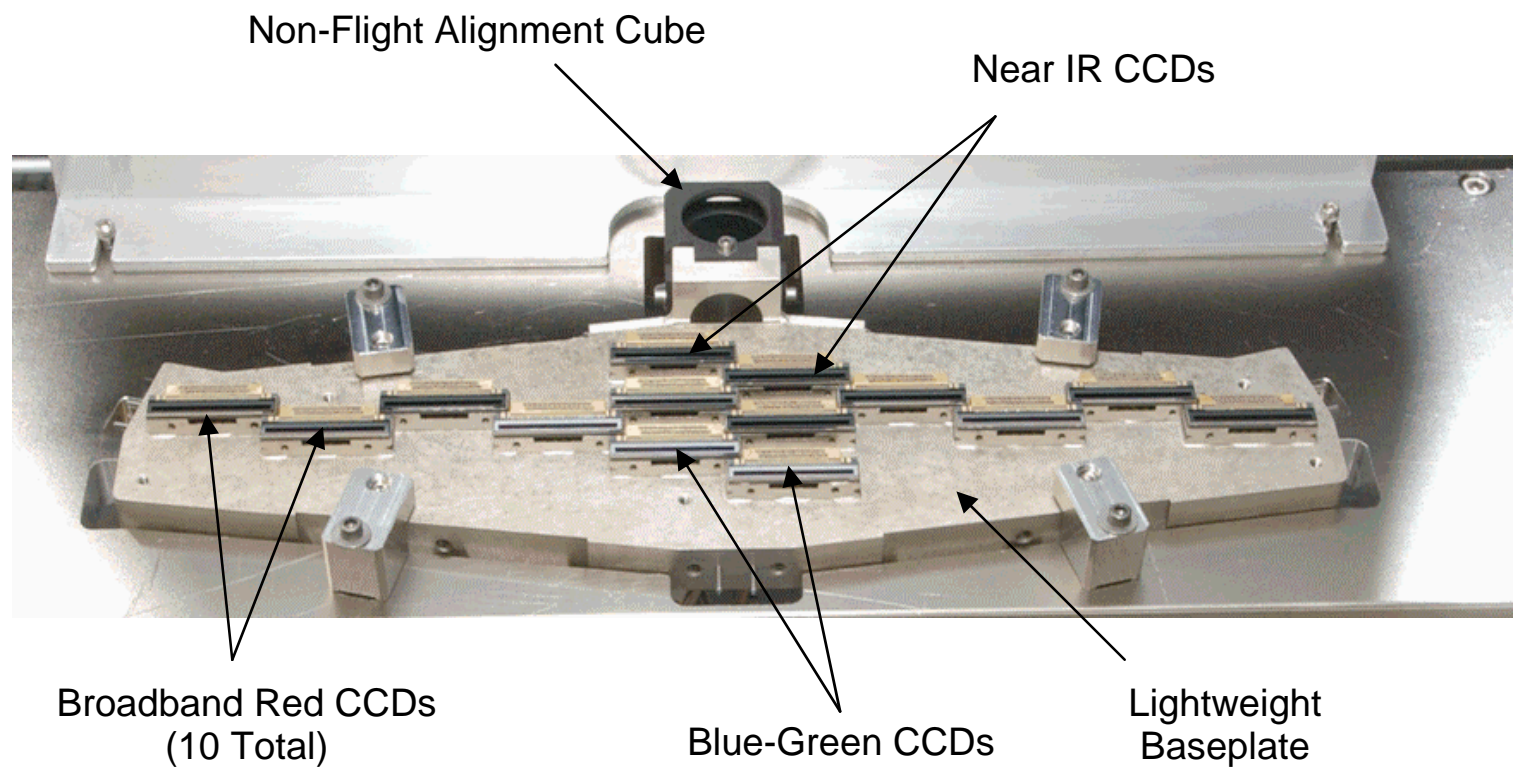

Figure 3: A Photograph of the HiRISE focal plane. 


\section{Optical Alignment}

The optical integration of the HiRISE optical system began with the installation of the primary mirror on its three point flexure mount. First the primary mirror was installed, then the secondary mirror. The optical alignment of the HiRISE optical system was complicated by the sensitivity of the F/1.73 primary to the secondary mirror. De-center and tip/tilt requirements for this pair of optics were $<12 \mu \mathrm{m}$ de-center and $<5$ arcseconds relative tilt of their respective optical axes. An alignment fixture was designed to perform the alignment of the secondary to the primary mirror. Because of the tight decenter tolerance, it was decided to align the primary and secondary mirror in a vertical fixture we called the "Cassegrain test fixture". The fixture is used because the primary and secondary mirror were tested like a Cassegrain telescope optic set. Figure 4 shows a photo of the test fixture. The best on-axis focus of the Cassegrain optic pair with the primary and secondary set to the HiRISE design spacing requirement was found to be $<0.02$ wave RMS most of the error was spherical aberration. A tooling sphere was placed to within 50 microns axially and along focus of the design Cassegrain focus, including for gravity and moisture effects on the structure. An optical flat at the top of the setup was aligned perpendicular to the primary optical axis to $<4$ arc-seconds via a set of penta-prisms, theodolites, and the optical reference surface on the back of the primary mirror. With the optics is this configuration the only unknown was the secondary mirror alignment. A Phasecam interferometer (4D Technologies) was aligned to null off the tooling sphere. Figure 4 shows where the Phasecam was located, along with a fold mirror that was used to reduce the height of the setup and keep the Phasecam orientation horizontal. The sphere was removed with out disturbing the Phasecam alignment and the interferometer light was allowed to double pass through the Cassegrain system. The secondary was then aligned to minimize power, coma and astigmatism. With the mounted primary mirror facing up in the vertical position, gravity distorted the primary's surface to $1 / 2$ peak-to-valley in the form of trefoil distortion. IntelliWave software was used to reduce the Phasecam data. Zernike coefficients were fit to each wavefront map to track and verify alignment of the Cassegrain system with alignment fidelity of less than 0.01 wave RMS, even with the large trefoil error present. 


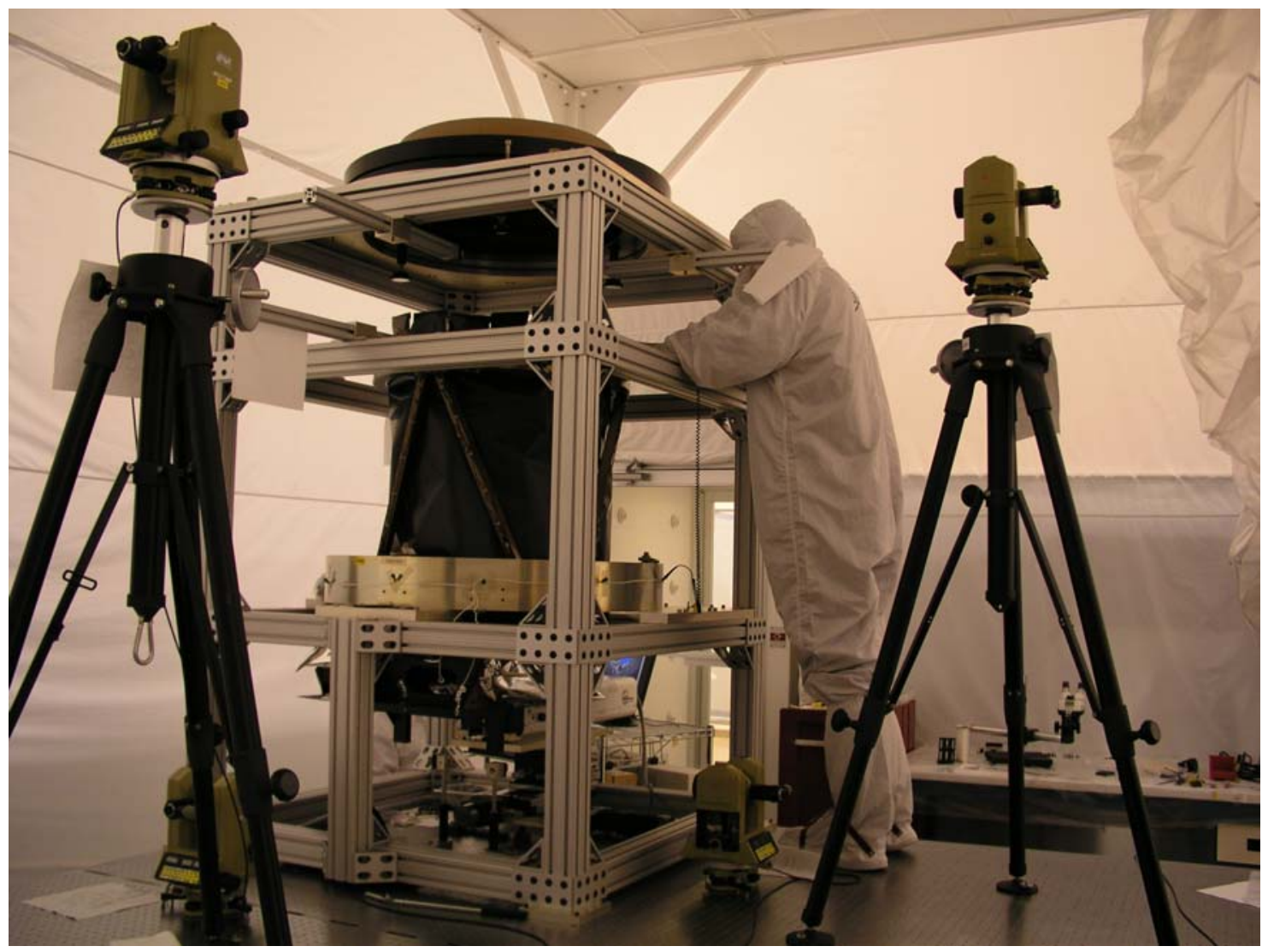

Figure 4: Cassegrain vertical test fixture with HiRISE inside. The theodolites that were used to view the penta-prisms are also visible

The alignment tolerances for fold mirrors \#1, \#2, and the tertiary mirror were such that the Axyzs coordinate measuring system was used to install the remaining optics via metrology. The Axyzs system consisted of at least two theodolites for 3-space position measurements and at least two theodolites for tip/tilt measurements. For the tertiary mirror, as many as five theodolites were used at one time during alignment. Each optic had precision fiducials and reference surfaces put on them (at the time of their manufacture) so the optical vertex could be located to within $\pm 50 \mu \mathrm{m}$ axially and along focus and the optical axis located to less than 15 arcseonds tip/tilt. The field stop and Lyot stop were also located using the Axyzs coordinated measuring system. The optical components were located with an updated optical raytrace that included the as-built optical radii. The location of the fold mirror \#2 was the compensator for the as-built optics. This mirror is also on a focus mechanism that provides the ability to move the mirror $\pm 5 \mathrm{~mm}$ from its nominal setting to fine tune HiRISE focus after launch.

\section{Interferometric testing}

After integration of the optics, a Focal Plane Array Optical Simulator (FPAOS) was placed on HiRISE to perform interferometric testing. The FPAOS consisted of a flat plate that mounts to HiRISE in a manner exactly like the flight FPA. The FPAOS had small convex lenses mounted to its surface where the vertices of the lenses' radii coincide with 
the nominal FPA focal surface. The convex surfaces were coated with aluminum so they reflected the beam back through the optical system for double pass interferometry. Small $1 \mathrm{~cm}$ diameter tooling spheres were also mounted to the FPAOS surface at positions that were carefully measured to perform boresight metrology across the focal plane. The interferometry from the small spheres was poor, but for finding the interferometer null to measure boresight the small spheres was adequate. Figure 5 shows a diagram of the FPAOS.

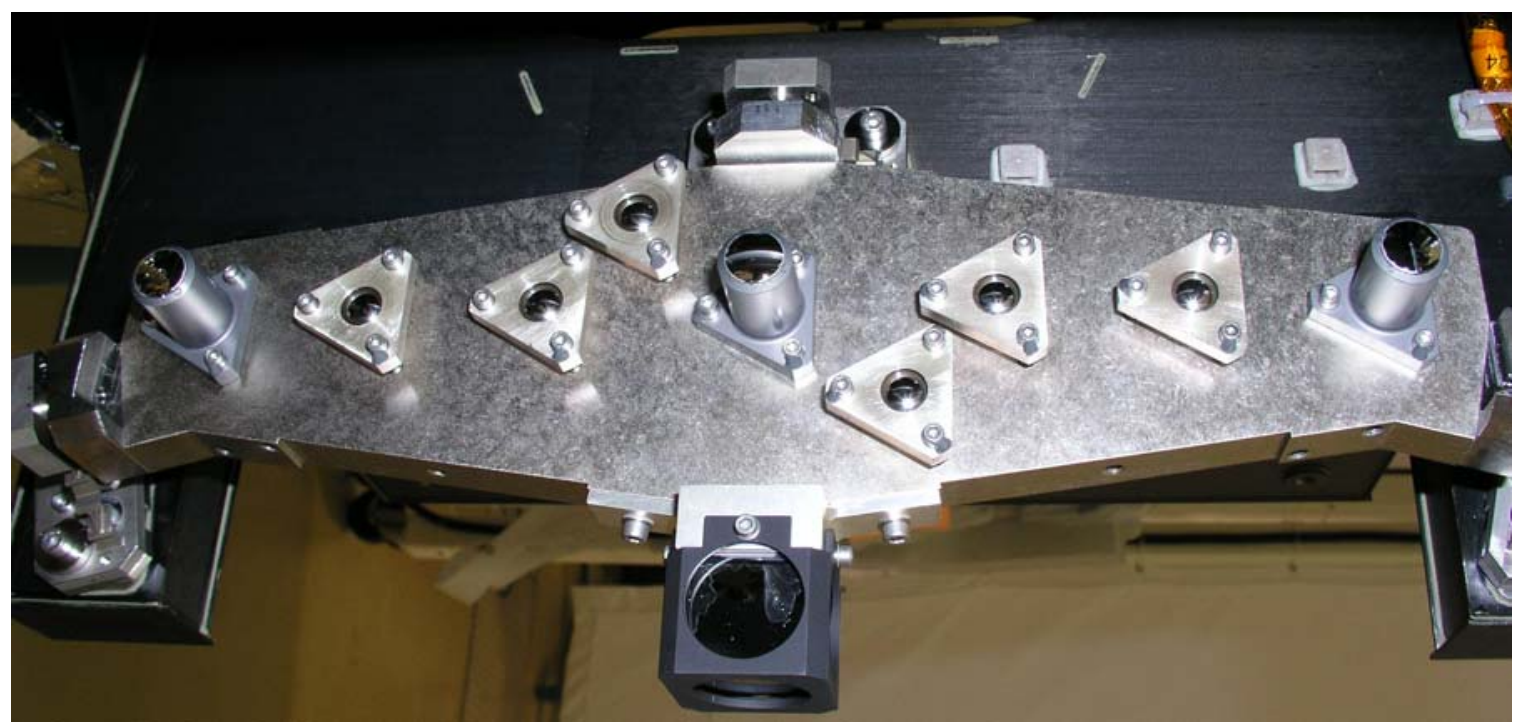

Figure 5: FPAOS showing the three coated convex lens surfaces and the six tooling balls.

Testing of HiRISE by interferometer was performed in Ball Areospace's Test Station \#2 facility. The test station uses a 0.75 meter diameter 4.0 meter EFL off-axis parabola to feed collimated light to HiRISE. The Phasecam was at the focus of the off-axis parabola. The interferometer setup was in double pass mode where the return beam is reflected from the coated convex lens surfaces on the FPAOS. Figure 5 shows a diagram of the Tests Station \#2 setup. 


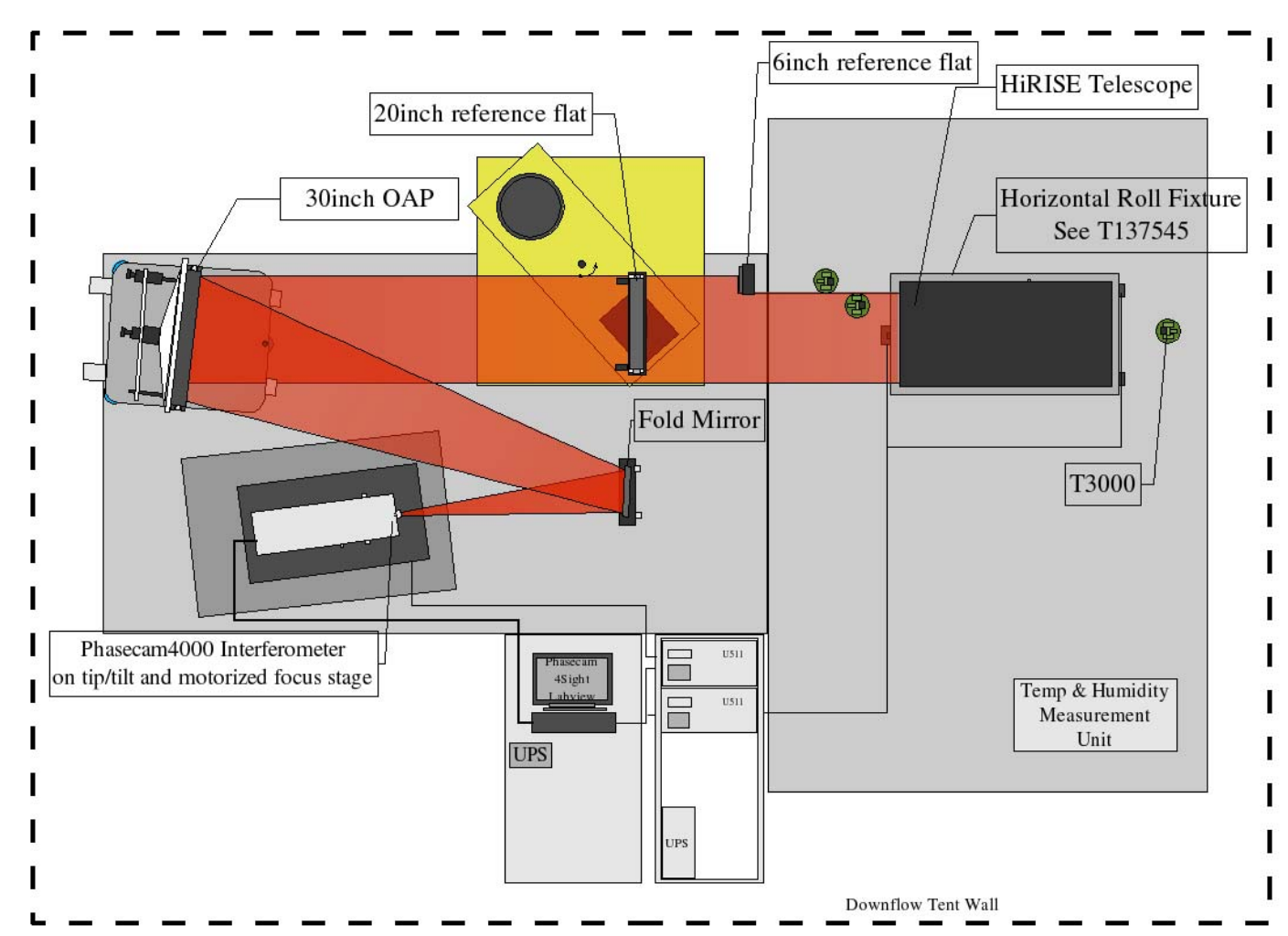

Figure 6: Balls Test Station \#2 facility showing HiRISE under test.

Because of the very light weight HiRISE structure the secondary mirror was de-centered from its nominal position by approximately $30 \mu \mathrm{m}$, or about three time the de-center requirement. To determine the Zero-G optical performance of HiRISE, the telescope was rotated 180 degrees about its boresight and the two surface maps were averaged using Intelliwave software to determine the resultant zero-G wavefront. The gravity compensated wavefront achieved was $\sim 0.045$ waves RMS (633nm) across the full field.

The flight focal plane array was then installed on to HiRISE using the wave front data to determine the best position for the flight focal plane. Imaging tests were then performed on the HiRISE optical system

\section{Optical imaging testing}

The imaging performance of HiRISE was again tested in the Ball's test station \#2 facility. To determine the exact focus of the HiRISE system, the test station was first collimated using a 0.5 meter optical flat. The focus from the Phasecam was passed through a pinhole during interferometric auto-collimation of the test station. A diffuse reflector was then placed behind the carefully set pinhole to send white light through the aligned test station. Images were collected with HiRISE of the point like image of the pinhole to determine the best focus position for the focus mechanism. Various targets were placed at the pinhole's position, pinhole removed, to aide in determining the imaging performance of the HiRISE optical system. A magnetic mount was used to hold the other targets at the 
same focus location as the pinhole. Figure 7 shows an image taken with HiRISE of a 2000 Line/inch bar target at the focus of the collimator. Each bar pair would represent a $\sim 1$ meter object at a $300 \mathrm{~km}$ distance. The plot across the bars shows that the HiRISE MTF is on order $20 \%$ for one meter scale objects at $300 \mathrm{~km}$. This demonstrates that HiRISE is clearly meeting its requirement that it must be able to detect 1 meter scale objects on the surface of Mars from a $300 \mathrm{~km}$ orbit.

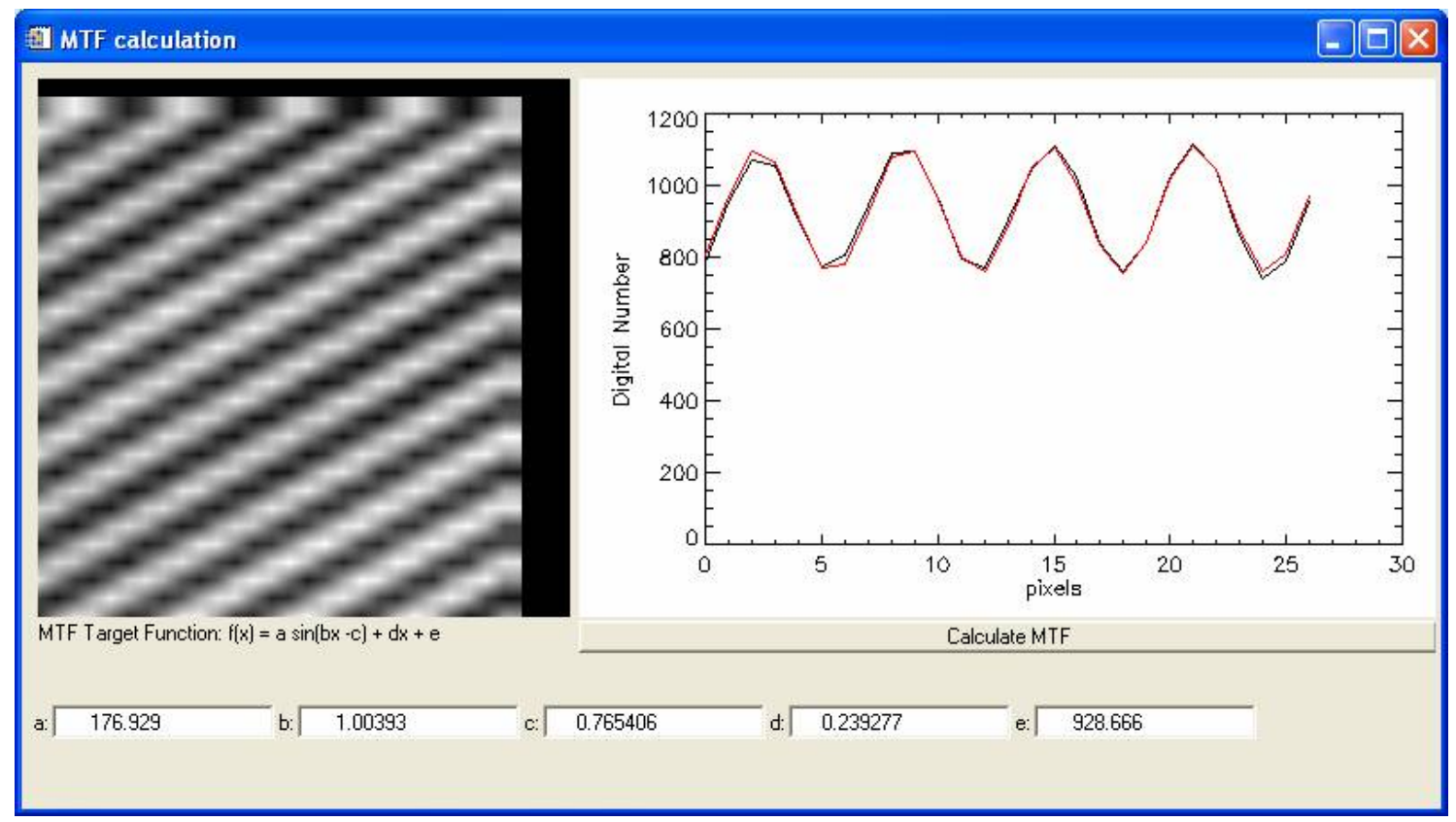

Figure 7: Image of a 2000 Line/inch target at the focus of the 4 meter EFL collimator. The plot to the right shows an intensity plot across the bar pattern.

\section{Conclusion}

HiRISE will launch on the MRO spacecraft in early August 2005. The optical alignment techniques described in the paper allowed building of the HiRISE optical system in a methodical approach without the need for many alignment iterations. The optical performance of HiRISE, as verified by test, appear to meet the HiRISE requirement of being able to detect one meter objects ( 20\% MTF) on the surface of Mars from orbit. We would like to thank Roger Johnston, of LightWork Optics, for his help in designing the "Vertical Cassegrain Test".

\section{REFERENCES}

[a] J.W. Bergstrom, et al, "MRO High Resolution Imaging Science Experiment (HiRISE): Instrument Test, Calibration and Operating Constraints", 55th International Astronautical Conference, Vancouver, BC, Canada, Oct 2004.

[1] Zurek, R., "Mars Exploration Strategy: Follow the Water,” Keynote Presentation, 24th Annual AAS Guidance and Control Conference, Breckenridge, CO, Feb 2001. 
[2] A S McEwen et al, "MRO High Resolution Imaging Science Experiment (HiRISE): Science Expectations”, Sixth International Conference on Mars, Pasadena, CA, July 2003.

[3] S.W. Lee et al, "Mars Reconnaissance Orbiter Design Approach for HighResolution Surface Imaging”, 26th Annual AAS Guidance and Control Conference, Breckenridge, CO, Feb 2001.

[4] D. Dorn, et al, "HiRISE Focal Plane for Use on the Mars Reconnaissance Orbiter”, Proceedings of the SPIE , Vol. 5167, July 2003. 